\title{
Model for collaborative and adaptive multimedia content delivery in a collaborative m-learning environment
}

\author{
Aleksandar Karadimce, Danco Davcev \\ Faculty of Computer Science and Engineering \\ University Ss Cyril and Methodius - Skopje \\ Skopje, Republic of Macedonia \\ akaradimce@ieee.org,danco.davcev@finki.ukim.mk
}

\begin{abstract}
Smart mobile devices are already used for everyday communication between people. They have become ubiquitous devices for receiving and sharing important information, but they poses limited capability of storing and processing multimedia content. Considering that mobile devices will always have these mentioned limitations, the proposed solution is to use collaborative, adaptive multimedia content delivery and the cloud computing technology. In order to gain increased perception of quality of m-learning systems, the proposed model delivers three services for selection, transcoding and authoring multimedia content, which should be hosted by SaaS cloud computing model. The proposed model for collaborative and adaptive multimedia content delivery will provide the mobile learners with cloudbased services. These services will facilitate the delivery of the preferred multimedia content across heterogeneous network to various types of mobile devices that learners are using. The advantage of the proposed framework is proved by using a sample mobile application for our case study, which estimates the operation of the service for multimedia content selection and authoring service.
\end{abstract}

Keywords- collaborative m-learning, cloud computing, mobile devices, adaptive multimedia content delivery, QoS, QoE

\section{INTRODUCTION}

Today we see that progressively more people for everyday communication are using smart mobile devices, with this term we will identify smart phones and tablet PCs. These devices have become ubiquitous devices for receiving and sharing important information simply by using voice, SMS, MMS, email, Bluetooth and other push notification services. Mobile devices possess capability of storing and processing definite multimedia information by using the built-in camera and multiple network connections, such as Wi-Fi, 2G, 3G UMTS or 4G (LTE). Recently, there is tremendous growth of sensor technology for smart mobile devices like the accelerometers to detect the orientation of the device, GPS capabilities and gyroscopic sensors for navigation. Embedding all of this technology into a single mobile device makes in perfect instrument for social networking, travelling, electronic payment, distance learning, video conferencing and etc. Despite all of these capabilities of mobile devices, they have certain limitations for memory, processing and battery duration that is an emerging research challenge.

Using a single device for receiving and sharing information or storing and processing multimedia content or simply harnessing the sensor capabilities with limited power source is overloading the mobile device. Therefore, it is becoming increasingly more difficult to pack more processing power and storage in a small device and simultaneously keep the battery power affordable. Bearing in mind that mobile device will always have these mentioned limitations, the proposed solution is to find a way how to offload the heavy memory storage and high performance processing tasks [1], [2]. The process of offloading refers to a technique that is used to overcome the limitations of mobile devices in terms of computation, memory and battery [3], [4]. Strongly related to offloading concept are mobile computing (MC) [5] and the technological paradigm cloud computing (CC) [6]. If we consider them together, today they are recognized as mobile cloud computing (MCC), which refers to an infrastructure where both the storage and the data processing happen outside of the mobile device from which an application is launched [6]. Because of the heterogeneity of the usage environment, the learner's multimedia content cannot be delivered to the receiving users according to their QoS capabilities. The challenge is to define a collaborative $\mathrm{m}$ learning environment that will consider the individual's learning context for delivering the multimedia content to mobile devices with heterogeneous capacity and capability. Therefore, we are proposing model for collaborative, adaptive multimedia content delivery in collaborative m-learning environment that can be used by hybrid mobile devices. The proposed model will provide extensive analysis of the layered Quality of Experience (QoE) framework that explains the relation between the influencing factors that affect the usage of the appropriate adaptation service.

This paper is organized as follows: Section II gives an overview of the related work. Section III presents our proposed adaptive collaboration algorithm based on QoS and QoE estimation. Section IV presents the results considering the case study for the proposed services used in collaborative mlearning system. Finally, Section V concludes the paper and proposes future work. 


\section{RELATED WORK}

Since, mobile smart devices have small computing power and work on a low network bandwidth there is a need to use transcoding technology in order to adapt the original streaming media for mobile environments. Considering that wireless networks have variable bandwidths depending on the movement of clients away from Access Point, this makes it hard to support constant stable QoS streams for mobile users. Therefore, some of the existing researches have proposed an algorithm that provides seamless streaming media services based on the network adaptive bit rate control and reduces transmission failure, by using transcoding bit-rate decision algorithm is proposed to provide stable QoS streams for mobile users [7]. Others have proposed frameworks that are using a QoS selection algorithm for finding the best sequence of adaptation services in order to maximize the user's satisfaction with the delivered content, considering only the influence of QoS factors, such as jitter, delay and packet loss [8]. They provide dynamical adaptation of the content based on the size and format of multimedia contents with consideration to the QoS context-aware conditions.

The static content adaptation generates and stores different variants of the same content on a content server, creating multimedia contents that are formatted for a certain mobile device or class of devices. Typical example of static content adaptation is HTTP Adaptive Streaming approach, which encodes and stores multiple versions of each single video at different bitrates, and let the client choose instant by instant the best suitable version to download [9]. However, the dynamic content adaptation is transcoding from one format to the other only in the moment of request. The transcoding process in dynamic content adaptation depends from the location where it takes place, and is been classified into three categories: serverbased, client-based, and proxy-based [7]. Reviewing the previously mentioned m-learning systems we conclude that the key components of a context-aware mobile educational content adaptation mechanism are the input (learner's mobile context), the adaptation engine and the output (the adapted multimedia educational content). Also, we have concluded that most of the existing frameworks for media content adaptation that have been analyzed by their properties for different media types (video, voice, images and text) are requiring different adaptation algorithms.

Education based on the mobile cloud has been introduced as a novel state of the art education in the area of intelligent learning and researchers have developed theoretical model framework for cloud-based intelligent learning to guide the design and development of an intelligent mobile cloud education system [10]. The proposed theoretical model is using an intelligent engine to create personalized learning plan for the users that can be adjusted in time-manner based on learners' feedback and outcomes [10]. The collaborative multimedia environment has to overcome the challenges of heterogeneity where the framework has to support service selection that satisfies context-aware QoS needs [11]. This means that for our proposed model for adaptive multimedia content delivery we have to take into considerations different influencing factors in order to create an intelligent collaborative mlearning environment and content.

\section{ADAPTIVE COLLABORATION ALGORITHM BASED ON QOS AND QOE ESTIMATION}

The framework proposes layered mapping of impact from user estimation, context-aware conditions and technological capabilities that affect the process of multimedia adaptation in collaborative m-learning environment. A prerequisite for efficient adaptation of multimedia information is a careful analysis of the properties of different media types. The process of estimating the learners' preferences requires collaborative gathering information that is relevant to the user in order to be able to deliver appropriate multimedia content. This opened opportunity to start considering aspects and influences that are relevant for the mobile learner, which requires extending the network connection QoS factors with the QoE. The QoE means overall acceptability of an application or service, as perceived subjectively by the end-user and represents multidimensional subjective concept that is not easy to be evaluated [12]. Therefore, we need to consider that besides previously analyzed network related QoS factors there are number of extended influencing factors that affect the mobile learners' experience. Clear understanding of different type of influencing factors can be observed if we recognize the difference between the QoS and QoE estimation (see Figure 1).

The collaborative adaptation uses original multimedia file from the centralized multimedia learning repository. In the first phase, for every user that participates in m-learning process, user cognitive style estimation is conducted using the VVLSR questionnaire [14]. Considering that the media files are initially highly dependent from the context-aware network conditions and the type of mobile device that the student is using, the multimedia content needs to be adapted according to the existing bandwidth of the context aware network connection, measured by QoS. In fact, we have used the current Available Network Adaptive Transcoding (ANAT) Algorithm in order to estimate the available network bandwidth [16]. The third category of system influencing factors should provide means for adaptation of multimedia contents for compatibility with different mobile devices. This process requires discovering the mobile device operating system, supported media format types and supported encoding preferences using the Wireless Universal Resource FiLe (WURFL) [17]. The last task is the authoring service (AS), which is a selective process where multiple pieces of multimedia data are combined into a complete presentation or service. Also, the AS should consider the technical capabilities of the learner's mobile device.

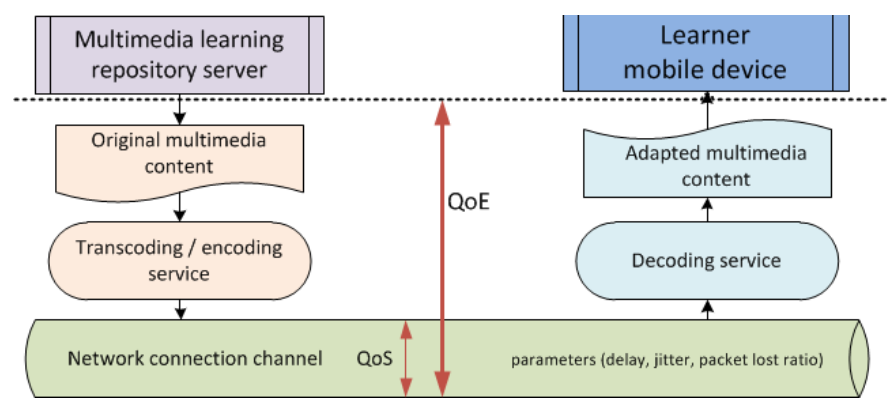

Figure 1. QoS and QoE aspects for adaptive content delivery 
The multimedia content delivery collaborative adaptation algorithm is following the previously established layered framework that considers QoS and QoE estimations. This algorithm is service-oriented content adaptation, which means that each module of the algorithm is dedicated to the appropriate service. Fundamentals of this proposed multimedia content delivery adaptation algorithm has been published previously as adaptation algorithm framework in [13]. There we have identified that the adaptation process can be carried out in a number of adaptation steps and there could be several collaborative adaptation services which execute each adaptation task that leads to different service composition possibilities [13]. The algorithm's pseudo-code is provided in Algorithm 1. Our proposed adaptive algorithm uses as input the available multimedia content, the answer received from VVLSR questionnaire [14], the response from WURFL request and estimation of network connection. The output from the algorithm is adapted multimedia content ready to be delivered to mobile leaners, Adapted (multimedia content). The algorithm is divided into three parts.

The first part is the service for cognitive estimation of user learning capabilities and it receives response from the user that has answered on the VVLSR questionnaire. Depending of the response value ( -3 to 3 ), the initialization of the appropriate index value is prepared. For this purpose we have used the conditional branching command switch, which provides commands for each and every case. In this way, users that have provided negative response on the VVLSR questionnaire are Verbalizers, which initializes the weight factor for text and audio content Sc. The second part of the algorithm is the network estimation service that provides optimal bandwidth selection for delivering multimedia content. Firstly, we are conducting real estimation (real bandwidth) of the network parameters using the ANAT algorithm [16] for the available network bandwidth. The weight factor for the optimal bandwidth ( $W_{\text {bandwidth }}$ ) is calculated as minimum from the real bandwidth estimation and the supported mobile device bandwidth divided with the available real bandwidth. The network estimation provides service for transcoding the multimedia content based on the existing context-aware network conditions. The last part of the algorithm is the device estimation service that should help to adapt the multimedia content for the type of device. The process of estimating the technical capabilities of the end user device is based on the information received from the WURFL request [17]. The first task is to estimate the type of device that the learner is using. The WURFL request provides information for the display of mobile device, which help us define the weight factor $\left(W_{h{ }^{*}}\right)$ for ration between the display height and width. Depending of the device type, we have anticipated tree different mobile OS (Android, iOS and Windows Mobile). For every case of mobile OS, we should provide method of conversion of the multimedia content in appropriate format. For example, if the learner uses mobile device with Android mobile OS, and the audio content is not in any of the supported formats (AAC, MP3, MIDI, OGG or WAV), it should be converted. Appropriate method of conversion is applied for the image and video content. The weight factor for encoding $\left(W_{\text {enc }}\right)$ is calculated as minimum of encoding index for text, audio, image and video content.

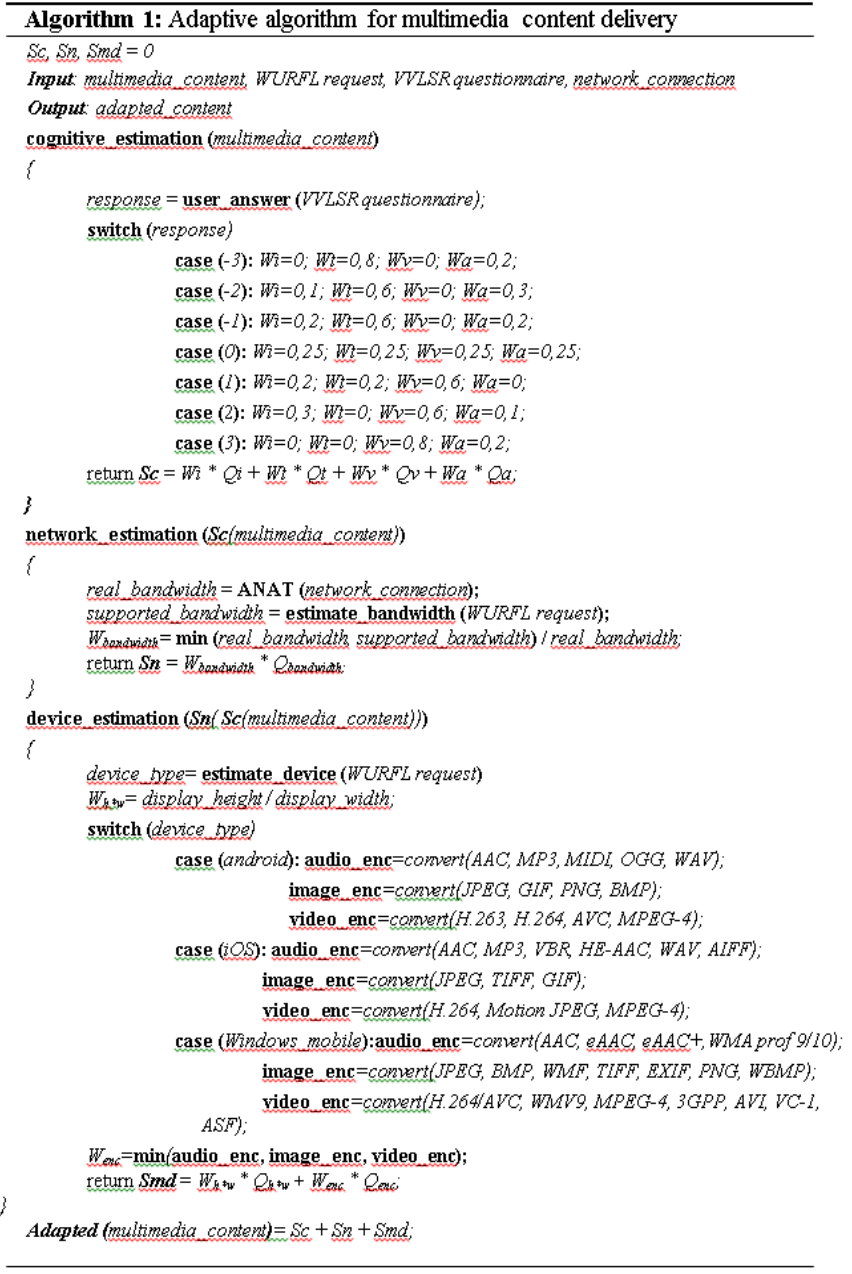

It is significant to mention that the order of calling the adaption services is also very important, because it is following the proposed layered framework for mobile multimedia content adaptation. Previously mentioned three services are correlated between them, they provide selection, transcoding and authoring of the multimedia content. These services, as integral part of the collaborative multimedia content delivery adaptation algorithm, require high processing capabilities. Therefore, their implementation should be done using the SaaS cloud-based model. Considering the processing power that is needed for the adaptation process and the large storage for centralized multimedia learning repository this QoE framework is based on the mobile cloud computing platform. These different services used in the collaborative adaptation process have been observed in detail by the proposed case study.

\section{CASE STUDY FOR THE PROPOSED COLLABORATIVE ADAPTATION SERVICES}

Another major challenge that m-learning systems are facing is how to adapt multimedia contents in order to be compatible with different mobile devices. Possible solution was to use the WURFL, which is a software component that maps HTTP Request headers to the profile of the HTTP client (Desktop, Mobile Device, Tablet, etc.) that issued the request [17]. This software component is able to detect learner's mobile device 
technical capabilities during the initial HTTP request and compare it with existing devices from device description repository (DDR) [17]. Using the information gathered by the WURFL component we will be able to make collaborative adaptation of the multimedia content to the mobile device supported capabilities (device type, browser or screen size). This way the WURFL model of detection is appropriate for discovering the technical capabilities of mobile device. The advantage of the proposed QoE framework is elaborated in the following m-learning case study. First time the learner is connected to the m-learning system, it is conducted on authorization to the system. During this process of authorization we are able to conduct estimation what are the technical capabilities of mobile device. This can be done by sending a WURFL request. Second process that should be conducted is the collaborative estimation of cognitive learning style. This will help us to create the user profile of the mobile learner, what type of multimedia contents he prefers to receive. Advantage of the proposed approach is to provide the mlearning system with estimation of QoS network parameters and the cognitive style estimation of the learner.

For the purpose of this case study we have created sample mobile device application called MCloud-mobile client. This application is intended for teaching the students how to create or drop database tables. Student after selecting the learning topic, the application MCloud-mobile client is asked to confirm in a collaborative way the learning style preference. The estimation of cognitive learning style in this case study has been implemented as learner's choice using three buttons for Verbalizer, Visualizer and Bimodal users. With the selection of his preferred cognitive learning style students are presented with different multimedia content. The mobile application for students that have selected Verbalizer as preferred learning style is presenting only with SQL syntax for creating database table, see Figure 2 (a). The students that are equally comfortable with both cognitive learning styles are presented with a mix of multimedia content, text and images, see Figure 2 (b). The MCloud-mobile client is able to provide the student with direct information based on their section.

\section{CONCLUSION AND FUTURE WORK}

The quality of perceived learner's experience is increased when the multimedia contents is adapted to the learners preferred style, mobile device capabilities and context-aware conditions in a collaborative way. The proposed model for collaborative adaptive multimedia content delivery in $\mathrm{m}$ learning environment provides extensive analysis of the layered QoE framework that explains the relation between the influencing factors that affect the usage of the adaptation service. The case study demonstrates that every student is able to see his expectation of multimedia learning content based on its preferred selection in a collaborative way. In this way, we proved the efficiency and usability of our model for collaborative adaptive multimedia content delivery.

\section{REFERENCES}

[1] K. Kumar, J. Liu, Y.H. Lu, and B. Bhargava, "A Survey of Computation Offloading for Mobile Systems," Mob. Netw. Appl. 18, 1 (February 2013), pp. 129-140. DOI=10.1007/s11036-012-0368-0.

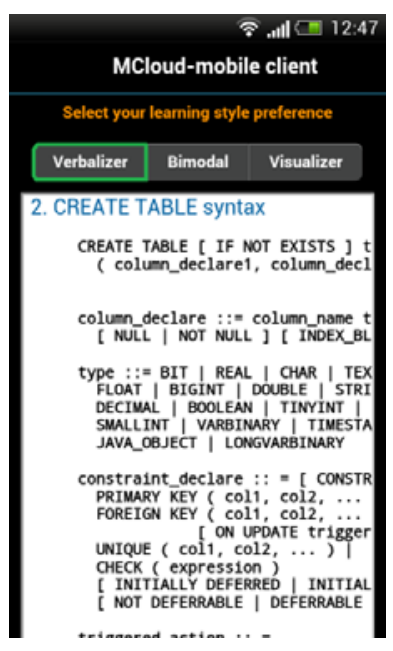

Verbalizer (a)

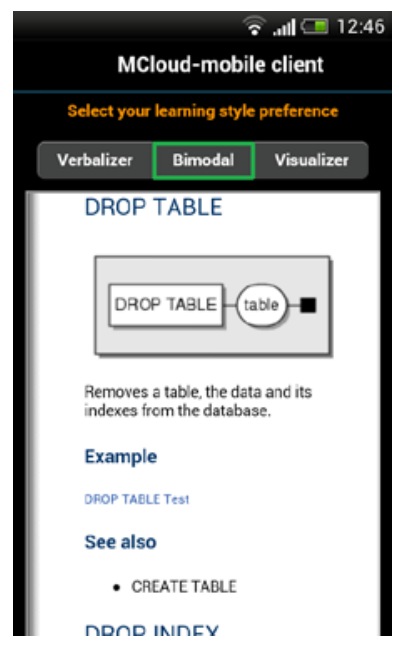

Bimodal (b)
Figure 2. Preferred learning style using the MCloud-mobile client.

[2] E. Lagerspetz and S. Tarkoma, "Mobile search and the cloud: The benefits of offloading," Proceedings in PerCom, pp. 117-122, 2011.

[3] D. Kovachev, R. Klamma, "Framework for Computation Offloading in Mobile Cloud Computing," International Journal of Interactive Multimedia and Artificial Intelligence (IJIMAI) 7(1), pp. 6-15, 2012.

[4] D. Kovachev, T. Yu, and R. Klamma, "Adaptive Computation Offloading from Mobile Devices into the Cloud," In Proceedings of the 2012 IEEE 10th ISPA '12. IEEE Computer Society, Washington, DC, USA, pp. 784-791. DOI=10.1109/ISPA.2012.115.

[5] M. Satyanarayanan, "Mobile computing: the next decade," In Proceedings of the 1st ACM Workshop on Mobile Cloud Computing \& Services: Social Networks and Beyond (MCS '10). ACM, New York.

[6] B. Sosinsky, Cloud Computing Bible (1st ed.). Wiley Publishing, 2011.

[7] Dongmahn Seo and Inbum Jung, "Network-adaptive autonomic transcoding algorithm for seamless streaming media service of mobile clients," Multimedia Tools Appl. 51, 3 (February 2011), pp. 897-912.

[8] K. El-Khatib, G. v. Bochmann, and A. El-Saddik, "A QoS-based Service Composition for Content Adaptation," In Proceedings of the 2007 IEEE 23rd International Conference on Data Engineering Workshop (ICDEW '07). IEEE Computer Society, Washington, DC, USA, pp. 331-338.

[9] V. Menkovski and A. Liotta, "QoE for mobile streaming," Published in Mobile Multimedia - User and Technology Perspectives, InTech Open Access Publisher, ISBN: 978-953-307-908-0, 2012.

[10] M. Wang; J.W.P. Ng, "Intelligent Mobile Cloud Education: Smart Anytime-Anywhere Learning for the Next Generation Campus Environment," 2012 8th International Conference on IE, pp.149-156.

[11] M.S. Hossain, "QoS in web service-based collaborative multimedia environment," Advanced Communication Technology (ICACT), 2014 16th International Conference on , vol., no., pp.881,884, 2014.

[12] F. Agboma, and A. Liotta, "Quality of experience management in mobile content delivery systems", Published in Springer 2010. pp. 85-98.

[13] A. Karadimce and D. Davcev, "Personalized Multimedia Content Generation Using the QoE Metrics in Distance Learning Systems," Proceedings of ADAPTIVE 2012. pp. 1-6, ISBN: 978-1-61208-219-6.

[14] R. E. Mayer and L. J. Massa: Three facets of visual and verbal learners: Cognitive ability, cognitive style and learning preference. Published in Journal of Educational Psychology, pp. 833-846, 2003.

[15] D. Davcev and A. Karadimce, "Model for interactive, collaborative and multimedia mobile learning environment," Proceedings of MoMM2013, 2-4 December, 2013, pp. 585-588, ISBN: 978-1-4503-2106-8

[16] D. Seo and I. Jung: Network-adaptive autonomic transcoding algorithm for seamless streaming media service of mobile clients. Multimedia Tools Appl. 51, 3, pp. 897-912, 2011.

[17] WURFL - Wireless Universal Resource FiLe, a Device Description Repository (DDR) by ScientaMobile. http://wurfl.sourceforge.net/ 